\title{
Sweets, sweetened beverages, and risk of pancreatic cancer in a large population-based case-control study
}

\author{
June M. Chan · Furong Wang · Elizabeth A. Holly
}

Received: 11 September 2008/ Accepted: 20 February 2009/Published online: 11 March 2009

(C) The Author(s) 2009. This article is published with open access at Springerlink.com

\begin{abstract}
Objective We examined the associations between sweets, sweetened and unsweetened beverages, and sugars and pancreatic cancer risk.

Methods We conducted a population-based case-control study (532 cases, 1,701 controls) and used multivariate logistic regression models to calculate odds ratios (OR) and 95\% confidence intervals (CI). Because associations were often different by sex, we present results for men and women combined and separately.

Results Among men, greater intakes of total and specific sweets were associated with pancreatic cancer risk (total sweets: OR $=1.9,95 \%$ CI: $1.0,3.6$; sweet condiments: $\mathrm{OR}=1.9,95 \%$ CI: $1.2,3.1$; chocolate candy: $\mathrm{OR}=2.4$, 95\% CI: 1.1, 5.0; other mixed candy bars: $\mathrm{OR}=3.3,95 \%$ CI: $1.5,7.3$ for $1+$ servings/day versus none/rarely). Sweets were not consistently associated with risk among women. Sweetened beverages were not associated with increased pancreatic cancer risk. In contrast, low-calorie soft drinks were associated with increased risk among men only; while other low-/non-caloric beverages (e.g., coffee, tea, and water) were unassociated with risk. Of the three sugars assessed (lactose, fructose, and sucrose), only the milk sugar lactose was associated with pancreatic cancer risk $(\mathrm{OR}=2.0,95 \% \mathrm{CI}: 1.5,2.7$ comparing extreme quartiles).
\end{abstract}

J. M. Chan ( $\square)$ · F. Wang · E. A. Holly

Department of Epidemiology and Biostatistics, University

of California San Francisco, San Francisco, CA, USA

e-mail: june.chan@ucsf.edu

J. M. Chan

Department of Urology, University of California San Francisco,

San Francisco, CA, USA
Conclusion These results provide limited support for the hypothesis that sweets or sugars increase pancreatic cancer risk.

Keywords Pancreatic cancer - Epidemiology · Sweets · Beverages

\section{Introduction}

Pancreatic cancer is the fourth leading cause of cancer death among men and women in the United States and the most fatal cancer. A number of 37,680 individuals were estimated to have been diagnosed with pancreatic cancer and 34,290 people were expected to die of the disease in 2008 [1]. Greater age, cigarette smoking, and male sex are fairly well-established risk factors for this deadly cancer. Diabetes also has been linked to greater risk of pancreatic cancer, and meta-analyses suggest that this link may be causal, or at least that metabolic conditions predisposing to diabetes precede pancreatic cancer [2, 3]. Four prospective studies that examined banked serum have linked higher fasting glucose levels with subsequent risk of pancreatic cancer several years later [4-7], and pre-diagnostic plasma C-peptide levels have been linked to greater risk of pancreatic cancer [8]. In vitro, insulin increases pancreatic cancer cell proliferation in a dose-dependent manner [9]. Hyperglycemia, insulin insensitivity, and hyperinsulinemia can lead to very high insulin exposure of the exocrine pancreatic cells, and this may play a role in pancreatic cancer development $[4,9]$.

Accordingly, it has been of interest to investigate dietary practices that may predispose to diabetes, hyperglycemia, or hyperinsulinemia. We examined sweets, sweetened beverages, and sugars as risk factors for pancreatic cancer 
in a large population-based case-control study. For comparison, we examined artificially sweetened, low-calorie, or non-caloric beverages (e.g., diet soft drinks, water, tea, and coffee) and pancreatic cancer risk. We hypothesized that intake of sweets, sugar, and beverages with sugar would be associated with an elevated risk of pancreatic cancer, based on their possible positive correlation with hyperglycemia and hyperinsulinemia; while artificially sweetened or low-calorie beverages would be unassociated with risk given their lesser effect on insulin response.

\section{Materials and methods}

\section{Study population}

Details of this study have been published previously [1023]. Briefly, between 1995 and 1999, men and women with incident adenocarcinoma of the exocrine pancreas were identified in six counties of the San Francisco Bay Area (in-area cases) using rapid case ascertainment operated by the Northern California Cancer Center. Pancreatic cancer diagnoses were confirmed by contacting the participants' physicians and using Surveillance, Epidemiology, and End Results (SEER) abstracts. Eligible in-area cases were 2185 years of age, residents of one of the six counties, alive upon first contact, and could complete an in-person interview in English. Sixty-five eligible out-of-area cases also were interviewed. They were seen in the University of California San Francisco clinics and met the same criteria as in-area cases except that most were residents of counties adjacent to the six Bay Area counties. Of 798 eligible cases, 532 (67\%) completed an interview for this study and $8 \%$ refused to participate. The main reason the remaining eligible cases did not participate was because they died prior to contact. Control participants were frequency-matched to cases by sex and age within five-year categories and were selected from the target population using random digit dial. Controls older than 65 years were supplemented by random selection from Health Care Finance Administration lists (now the Centers for Medicare \& Medicaid Services). Of 2,525 eligible controls, 1,701 (67\%) completed an interview for this study. No proxy interviews were conducted. This study was reviewed and approved by the University of California San Francisco Institutional Review Board and written informed consent was obtained from all the participants prior to interview.

Assessment of intake of sweets and non-alcoholic beverages

Participants reported, via in-person interviews, their frequency of intake of individual food items that were listed on a 131-item food questionnaire for the time period of one year before their cancer diagnosis for cases, or interview for controls. Intake of seasonal food was averaged throughout the entire year. This semi-quantitative food-frequency questionnaire was obtained from the Harvard University, has been validated in a variety of populations [24-26], and is considered to perform similarly to the National Cancer Institute Diet History Questionnaire and the Block foodfrequency questionnaire [26]. In a validation study from the Nurses Health Study, the correlation coefficients between questionnaire and diet record responses for several of the sweet and beverage items of interest were: chocolate 0.41 , sweets 0.63 , coffee 0.77 , tea 0.86 , coke/pepsi 0.84 , and noncola carbonated beverages 0.40 [27].

The specified portion size of each food item was considered as one serving, and the food frequency responses were transformed into servings per day. The options for frequency of food intake were: never, $<1 /$ month (rarely), 1-3/month, 1/week, 2-4/week, 5-6/week, 1/day, 2-3/day, $4-5 /$ day, and $6+/$ day. Consumption of total and individual sweets and beverage items was examined. Sweets included pure chocolate candy bars or packets of candy (e.g., M\&Ms), other mixed candy bars (e.g., Snickers/Milky Way/Reeses), candy without chocolate (e.g., mints, lifesavers), and sweet condiments (i.e., jams/jellies/preserves/ syrup/honey). Total carbonated beverages included those with sugar (i.e., Coke/Pepsi/other colas, caffeine-free Coke/ Pepsi/other colas, and other) and sugar-free types (i.e., lowcalorie colas, low-calorie caffeine-free colas, and other lowcalorie carbonated beverages such as diet 7-up, Fresca, diet ginger ale, etc.). Other sweetened beverages assessed included punch/lemonade/non-carbonated fruit drinks (not juice). Other non-caloric or low-calorie beverages assessed for comparison included plain water, herbal tea, non-herbal tea, decaffeinated coffee, and caffeinated coffee. While we did not have specific information regarding sugar added to tea or coffee, we were able to examine overall teaspoons of sugar added to food or beverages. The range of the correlation coefficients among all of the sweets was 0.09-0.41. The strongest correlation was observed between pure chocolate candy bars or packets of candy and other mixed candy bars, and the weakest association was found between sweet condiments and candy without chocolate. The range of the correlation coefficients among all of the beverages was $0.00-0.32$. The strongest correlations were observed for items such as Coke/Pepsi/other colas with sugar and other carbonated beverages (correlation coefficient $=0.32$ ) and low-calorie colas with other low-calorie carbonated beverages (correlation coefficient $=0.24$ ) .

The completed food frequency questionnaires were sent to the Harvard School of Public Health, Department of Nutrition for assessment of nutrient intake using their previously published methods [28]. Of interest to this 
report, nutrients assessed included total calories, and three sugars-fructose, sucrose, and lactose. These were examined in quartiles based on control group cut-points.

\section{Statistical methods}

Odds ratios (OR) and $95 \%$ confidence intervals (CI) were computed using unconditional logistic regression to estimate the relative risk (hereafter called risk) of pancreatic cancer. No substantial difference in ORs was observed when we included or excluded study participants with extremely low $(<500 \mathrm{kcal} /$ day $)$ or extremely high $(>3,500 \mathrm{kcal} / \mathrm{day})$ caloric intake, and these participants were retained in the final analyses. The linear trend test was performed using the Wald procedure. Potential confounding effects were investigated for total calories, sex, body-mass index (BMI), cigarette smoking, race, education, history of diabetes, physical activity, and other food groups. Total caloric intake was divided into quartiles based on the consumption among control participants by sex (cutpoints: men: $\leq 1,545 ; 1,546-1,925 ; 1,926-2,364 ; \geq 2,365 \mathrm{kcal} /$ day; women: $\leq 1,333 ; 1,334-1,695 ; 1,696-2,113 ; \geq 2,114 \mathrm{kcal} /$ day). BMI was categorized as $<25$ (normal), 25.0-29.9 (overweight), and $\geq 30$ (obese) $\mathrm{kg} / \mathrm{m}^{2}$, based on the World Health Organization criteria. Smokers were defined as participants who had smoked $>100$ cigarettes in their lifetime, or a pipe or cigar for at least once a month for $\geq 6$ months. Participants were classified as: never smokers; former cigarette smokers who quit smoking $>15$ years ago; former cigarette smokers who quit smoking 1-15 years ago; former smokers who quit within one year prior to diagnosis or to interview, and current cigarette smokers; and pipe and/or cigar smokers. Participants provided selfreported data on race (white, black or African American, Asian or Pacific Islander, or "other"); education ( $<$ highschool graduate, high-school graduate, 1-4 years college, and graduate work); history of diabetes (yes/no); and frequency of non-occupational physical activity (i.e., $30 \mathrm{~min}$ intervals performed $\leq 1 /$ month, $2-4 /$ month, $2-6 /$ week, or $1+/$ day). We also considered intake of other food groups in quartiles based on previous publications from this population (i.e., red meat, white meat, dairy, vegetable and fruit, eggs, fish, whole grain, and refined grain) $[11,19,20]$.

Stepwise logistic regression modeling (using $p=0.05$ ) was used to determine the variables that provided the bestfit among the potentially confounding factors. By these criteria, only total energy appeared to confound the relationships between sweets and beverages and pancreatic cancer risk. For comparability with other studies and our previous publications, we first present a parsimonious model in the tables that was adjusted for the matching factors of age and sex, and for total energy intake. We then present a full multivariable model additionally adjusted for the following putative confounding factors: BMI, smoking status, race, education, history of diabetes, physical activity, and other food groups. Adjustment for other food groups included mutual adjustment for all sweet and beverage risk factors of interest plus previously examined food groups $[11,19,20]$ (e.g., total sweets were adjusted for all sweetened and unsweetened beverage intake, as well as consumption of red meat, white meat, vegetables and fruits, eggs, fish, dairy, whole grains, and refined grains). The results for men and women are presented combined and separately. Because there was little difference between the parsimonious model and the fully adjusted model, we present only the full model for the results stratified by sex. To further consider the effects of confounding or effect modification on these results, we examined total sweets and carbonated beverages (with or without sugar) in multivariable models within strata of BMI (normal, overweight, and obese), exercise (monthly vs. daily exercisers), diabetes (yes/no), and smoking history (current, former, never). We considered stratifying BMI further, however, limited numbers made this infeasible (e.g., BMI $\geq 35: n=13$ cases/31 controls; BMI $\geq 40: n=3$ cases $/ 6$ controls).

All statistical tests were two-sided and considered statistically significant when $p<0.05$. Statistical analyses were conducted using SAS software 9.1 (SAS Institute, Inc., Cary, NC).

\section{Results}

Demographic and other factors in this large populationbased case-control study are presented in Table 1. Cases and controls were similar with regards to race, BMI, and history of diabetes; and the majority of participants were non-Hispanic whites. Cases were more likely to have a recent smoking history than controls, whereas controls were slightly more educated than cases.

There was some evidence for positive associations between consumption of total and individual sweets and risk of pancreatic cancer among men (for comparisons of $1+$ servings/day versus rarely or never, total sweets $\mathrm{OR}=1.9,95 \%$ CI: 1.0, 3.6; sweet condiments $\mathrm{OR}=1.9$, 95\% CI: $1.2,3.1$; chocolate candy OR $=2.4,95 \%$ CI: 1.1 , 5.0; other mixed candy bars $\mathrm{OR}=3.3,95 \%$ CI: $1.5,7.3$ ) (Table 2). Among women, there was overall less support for any association with total or individual sweets, although there was a positive trend for sweet condiments (Table 2). Candy without chocolate was unassociated with risk of pancreatic cancer.

To further examine the impact of foods high in sugar, we examined sweetened beverages and the risk of pancreatic cancer (Table 3). Overall, total sweetened beverages, including sugar-based sodas, colas, and non-carbonated fruit 
Table 1 Demographic characteristics of pancreatic cancer patients and control participants in a population-based case-control study in the San Francisco Bay Area, California

\begin{tabular}{llll}
\hline Characteristic & $\begin{array}{l}\text { Cases } \\
(N=532)\end{array}$ & & $\begin{array}{l}\text { Controls } \\
(N=1,701)\end{array}$ \\
\cline { 2 - 2 } & $n$ & & $n$
\end{tabular}

\begin{tabular}{|c|c|c|c|c|}
\hline \multicolumn{5}{|l|}{$\mathrm{Age}^{\mathrm{a}}$, years } \\
\hline$<50$ & 46 & 9 & 164 & 10 \\
\hline $50-59$ & 120 & 22 & 438 & 25 \\
\hline $60-69$ & 172 & 33 & 473 & 28 \\
\hline $70-79$ & 158 & 39 & 498 & 30 \\
\hline $80-85$ & 36 & 7 & 128 & 8 \\
\hline \multicolumn{5}{|l|}{ Sex } \\
\hline Men & 291 & 55 & 883 & 52 \\
\hline Women & 241 & 45 & 818 & 48 \\
\hline \multicolumn{5}{|l|}{ Race } \\
\hline White & 442 & 83 & 1,471 & 86 \\
\hline Black or African American & 46 & 9 & 78 & 5 \\
\hline Asian or Pacific Islander & 35 & 7 & 119 & 7 \\
\hline $\begin{array}{l}\text { American Indian or Alaskan } \\
\text { Native or other }\end{array}$ & 9 & 2 & 33 & 2 \\
\hline \multicolumn{5}{|l|}{ Hispanic ethnicity } \\
\hline Yes & 25 & 5 & 114 & 7 \\
\hline No & 507 & 95 & 1,585 & 93 \\
\hline \multicolumn{5}{|l|}{ Body mass index (WHO), $\mathrm{kg} / \mathrm{m}^{2}$} \\
\hline Normal, $<25.0$ & 280 & 53 & 993 & 58 \\
\hline Overweight, 25.0-29.9 & 197 & 37 & 552 & 33 \\
\hline Obese, $\geq 30$ & 52 & 10 & 147 & 9 \\
\hline \multicolumn{5}{|l|}{ Smoking } \\
\hline Never & 163 & 31 & 652 & 38 \\
\hline \multicolumn{5}{|l|}{ Cigarette } \\
\hline Former, quit $>15$ years ago & 133 & 25 & 508 & 30 \\
\hline Former, quit $1-15$ years ago & 89 & 17 & 260 & 15 \\
\hline Current or quit $<1$ year ago & 131 & 25 & 208 & 12 \\
\hline Pipe/cigar & 16 & 3 & 73 & 4 \\
\hline \multicolumn{5}{|l|}{ Education } \\
\hline$<$ High-school graduate & 71 & 13 & 162 & 10 \\
\hline High-school graduate & 164 & 31 & 372 & 22 \\
\hline $1-4$ years college & 200 & 38 & 754 & 44 \\
\hline Graduate school & 97 & 18 & 413 & 24 \\
\hline \multicolumn{5}{|l|}{ History of diabetes mellitus } \\
\hline No & 455 & 86 & 1,538 & 90 \\
\hline Yes & 76 & 11 & 161 & 10 \\
\hline
\end{tabular}

Numbers may not add to total number of participants due to missing values

a Age at pancreatic cancer diagnosis for cases or age at interview for controls

drinks were unassociated with risk of pancreatic cancer. Among women, the highest versus lowest intake of punch/ lemonade/non-carbonated fruit drinks was associated with a borderline statistically significant doubling in risk, but intermediate intake categories were inversely associated with risk. In contrast, some, but not all, low-calorie carbonated drinks were linked to about a 50-80\% higher risk of pancreatic cancer when comparing consumption of $1+$ servings/day versus rarely. These relationships were only statistically significant among men when we examined associations stratified by sex (sugar-free carbonated beverages $\mathrm{OR}=1.8,95 \% \mathrm{CI}: 1.1,2.8 ;$ low-calorie cola $\mathrm{OR}=1.8,95 \% \mathrm{CI}: 1.1,2.9)$.

For further comparison, we examined other low- or noncaloric beverages and risk of pancreatic cancer. Coffee (with or without caffeine), herbal tea, and water were not associated with risk of pancreatic cancer among men, women, or both sexes combined. Non-herbal tea was not associated with risk among women, but there was a positive trend among men only $(\mathrm{OR}=1.6,95 \% \mathrm{CI} 1.1,2.2$ for $1+$ servings/day versus $<1 /$ month; $p$-value trend $=0.02$ ). Teaspoons of sugar or artificial sweeteners added to foods or beverages was also unassociated with risk of pancreatic cancer (multivariate $\mathrm{OR}=0.7,95 \% \mathrm{CI}: 0.5,1.1$ for $\geq 3$ vs. 0 teaspoons of sugar per day; multivariate $\mathrm{OR}=1.3,95 \%$ CI $0.8-1.9$ for $\geq 1$ packet sweetener vs. none per day; adjusted for age sex, calories, BMI, race, education, smoking, diabetes, and exercise).

In order to further consider the effects of confounding or effect modification on these results, we examined total sweets and carbonated beverages in multivariable models within strata of BMI, exercise, diabetes, and smoking history (data not shown in tables). There was some suggestion that the positive association for total sweets was stronger or limited to those who were obese, infrequent exercisers, or current smokers; although, with the smaller numbers in the finer categories, results were not statistically significant. When comparing consumption of $1+$ servings/day of total sweets versus none, the risks of pancreatic cancer across strata of BMI were: obese, $\mathrm{OR}=3.2,95 \% \mathrm{CI}: 0.7,15$; overweight, $\mathrm{OR}=1.1,95 \%$ CI: $0.5,2.4$; and normal, $\mathrm{OR}=1.2,95 \% \mathrm{CI}: 0.7,2.1$. Similarly, across categories of smoking, the odds ratios of pancreatic cancer for $1+$ servings/day of total sweets versus none were: current smoker, OR $=1.8,95 \% \mathrm{CI}$ : 0.6, 5.4; former smoker, $\mathrm{OR}=1.2,95 \% \mathrm{CI}$ : 0.5, 2.9; and never smoker, OR $=1.1,95 \%$ CI: 0.6, 2.4. Lastly, for the same comparison by exercise the odds ratios were: infrequent exercise, $\mathrm{OR}=2.0,95 \% \mathrm{CI}: 1.0,4.0$ for and daily exercisers, $\mathrm{OR}=1.0,95 \% \mathrm{CI}: 0.3,2.7$. The previously observed elevated risk for sugar-free carbonated beverages also appeared to be limited to those who were obese (obese, $\quad \mathrm{OR}=2.6, \quad 95 \% \quad \mathrm{CI}$ : $0.9, \quad 7.7$; overweight, $\mathrm{OR}=1.5,95 \% \mathrm{CI}: 0.9,2.6$; and normal $\mathrm{BMI}, \mathrm{OR}=1.3$, 95\% CI: 0.8, 2.1), did not have diabetes (no diabetes, $\mathrm{OR}=1.6,95 \% \mathrm{CI}: 1.1,2.3$; yes diabetes, $\mathrm{OR}=0.9,95 \%$ 
Table 2 Odds ratios (OR) and 95\% confidence intervals (CI) for pancreatic cancer and consumption of sweets in a population-based casecontrol study, San Francisco Bay Area, California

\begin{tabular}{|c|c|c|c|c|c|c|c|c|c|c|c|c|}
\hline \multirow[b]{2}{*}{ Sweets ( 1 serving) } & \multicolumn{8}{|c|}{ Men + Women } & \multicolumn{2}{|l|}{ Men } & \multicolumn{2}{|c|}{ Women } \\
\hline & Case $n$ & $\%$ & Control $n$ & $\%$ & $\mathrm{OR}^{\mathrm{a}}$ & $95 \% \mathrm{CI}^{\mathrm{a}}$ & $\mathrm{OR}^{\mathrm{b}}$ & $95 \% \mathrm{CI}^{\mathrm{b}}$ & $\mathrm{OR}^{\mathrm{b}}$ & $95 \% \mathrm{CI}^{\mathrm{b}}$ & $\mathrm{OR}^{\mathrm{b}}$ & $95 \% \mathrm{CI}^{\mathrm{b}}$ \\
\hline \multicolumn{13}{|c|}{ Total sweets, servings/day } \\
\hline 0 & 54 & 10 & 183 & 11 & 1.0 & Ref & 1.0 & Ref & 1.0 & Ref & 1.0 & Ref \\
\hline$<1$ & 316 & 60 & 1,156 & 68 & 0.8 & $0.6,1.2$ & 0.9 & $0.6,1.3$ & 1.2 & $0.7,2.2$ & 0.6 & $0.4,1.1$ \\
\hline$\geq 1$ & 156 & 30 & 362 & 21 & 1.2 & $0.8,1.8$ & 1.3 & $0.8,1.9$ & 1.9 & $1.0,3.6$ & 0.8 & $0.4,1.4$ \\
\hline Trend- $p$ & & & & & 0.3 & & 0.1 & & 0.01 & & 0.5 & \\
\hline \multicolumn{13}{|c|}{ Sweet condiments ( 1 tablespoon) } \\
\hline$<1 /$ month & 106 & 20 & 428 & 25 & 1.0 & Ref & 1.0 & Ref & 1.0 & Ref & 1.0 & Ref \\
\hline $1-3 /$ month & 82 & 16 & 364 & 21 & 0.9 & $0.6,1.2$ & 0.9 & $0.6,1.2$ & 1.1 & $0.7,1.7$ & 0.8 & $0.5,1.3$ \\
\hline 1-6/week & 254 & 48 & 696 & 41 & 1.3 & $1.0,1.7$ & 1.5 & $1.1,1.9$ & 1.6 & $1.1,2.4$ & 1.5 & $1.0,2.2$ \\
\hline$\geq 1 /$ day & 84 & 16 & 212 & 13 & 1.4 & $1.0,2.0$ & 1.6 & $1.1,2.3$ & 1.9 & $1.2,3.1$ & 1.5 & $0.9,2.6$ \\
\hline Trend- $p$ & & & & & 0.007 & & 0.0004 & & 0.002 & & 0.02 & \\
\hline \multicolumn{13}{|c|}{ Pure chocolate candy bar or packet (1) } \\
\hline$<1 /$ month & 245 & 47 & 863 & 51 & 1.0 & Ref & 1.0 & Ref & 1.0 & Ref & 1.0 & Ref \\
\hline $1-3 /$ month & 131 & 25 & 439 & 26 & 1.0 & $0.8,1.3$ & 1.1 & $0.8,1.4$ & 1.3 & $0.9,1.9$ & 0.9 & $0.6,1.2$ \\
\hline 1-6/week & 122 & 23 & 355 & 21 & 1.1 & $0.8,1.4$ & 1.1 & $0.9,1.5$ & 1.2 & $0.8,1.7$ & 1.0 & $0.7,1.5$ \\
\hline$\geq 1 /$ day & 28 & 5 & 44 & 3 & 2.0 & $1.2,3.2$ & 2.0 & $1.2,3.4$ & 2.4 & $1.1,5.0$ & 1.7 & $0.8,3.7$ \\
\hline Trend- $p$ & & & & & 0.08 & & 0.06 & & 0.07 & & 0.5 & \\
\hline \multicolumn{13}{|c|}{ Candy without chocolate (1 pack) } \\
\hline$<1 /$ month & 316 & 60 & 1,080 & 63 & 1.0 & Ref & 1.0 & Ref & 1.0 & Ref & 1.0 & Ref \\
\hline $1-3 /$ month & 93 & 18 & 298 & 18 & 1.0 & $0.8,1.4$ & 1.1 & $0.8,1.4$ & 1.1 & $0.8,1.6$ & 1.0 & $0.7,1.6$ \\
\hline 1-6/week & 90 & 17 & 250 & 15 & 1.2 & $0.9,1.6$ & 1.1 & $0.8,1.5$ & 1.3 & $0.9,1.9$ & 0.9 & $0.6,1.5$ \\
\hline$\geq 1 /$ day & 27 & 5 & 73 & 4 & 1.1 & $0.7,1.8$ & 1.0 & $0.6,1.6$ & 1.5 & $0.8,2.9$ & 0.6 & $0.3,1.3$ \\
\hline Trend- $p$ & & & & & 0.2 & & 0.5 & & 0.09 & & 0.3 & \\
\hline \multicolumn{13}{|c|}{ Other mixed candy bar (1) } \\
\hline$<1 /$ month & 276 & 52 & 1,041 & 61 & 1.0 & Ref & 1.0 & Ref & 1.0 & Ref & 1.0 & Ref \\
\hline $1-3 /$ month & 119 & 23 & 382 & 22 & 1.1 & $0.9,1.4$ & 1.1 & $0.9,1.4$ & 1.4 & $1.0,2.0$ & 0.8 & $0.5,1.2$ \\
\hline $1-6 /$ week & 109 & 21 & 253 & 15 & 1.4 & $1.1,1.9$ & 1.4 & $1.0,1.8$ & 1.5 & $1.1,2.2$ & 1.2 & $0.8,1.9$ \\
\hline$\geq 1 /$ day & 22 & 4 & 25 & 1 & 2.7 & $1.5,4.9$ & 2.2 & $1.2,4.1$ & 3.3 & $1.5,7.3$ & 1.0 & $0.3,3.4$ \\
\hline Trend- $p$ & & & & & 0.0004 & & 0.005 & & 0.001 & & 0.7 & \\
\hline
\end{tabular}

a Adjusted for age, sex, and energy intake (kcal/day, men: quartiles, women: quartiles); ORs were not computed if cells had $<5$ observations

b Additionally adjusted for body mass index $\left(<25.0,25.0-29.9, \geq 30 \mathrm{~kg} / \mathrm{m}^{2}\right)$, race (white, black/African American, Asian/Pacific Islander, others), education ( $<$ high-school graduate, high-school graduate, college, graduate work), smoking (never smoker, former cigarette smoker who quit smoking $>15$ years ago, former cigarette smoker who quit smoking $1-15$ years, current cigarette smoker or quit $<1$ year ago, pipe, and/or cigar smoker), history of diabetes (yes, no) and physical activity (30-min moderate exercise each time: $<1$ month, 1-4/month, 2-6/week, and daily); total sweets was additionally adjusted for quartiles of total red meat, white meat, vegetable and fruit, eggs, fish, dairy, whole grain, and refined grain, and sweetened beverages $(0,<1$, and $\geq 1$ serving per day)

CI: 0.3, 2.6), or were current smokers (current, $\mathrm{OR}=2.6$, 95\% CI: 1.1, 5.9; former, $\mathrm{OR}=0.5,95 \% \mathrm{CI}: 0.2,1.3$; never, $\mathrm{OR}=0.8,95 \% \mathrm{CI}: 0.4,1.5)$.

We examined the risk of pancreatic cancer associated with intake of the sugars fructose, sucrose, and lactose (Table 4). Of these, only lactose was associated with an elevated risk of pancreatic cancer when comparing extreme quartiles. To address the possibility that this result for lactose (found in milk products) may have been confounded by fat intake, we further adjusted for total fat intake and results were similar (combined: $\mathrm{OR}=2.0,95 \%$
CI: $1.5,2.7, p<0.0001$; men: $\mathrm{OR}=2.1,95 \% \mathrm{CI}: 1.3,3.0$, $p=0.003$; women: $\mathrm{OR}=1.7,95 \% \mathrm{CI}: 1.1,2.7, p=0.03$, when comparing extreme quartiles, Table 4).

\section{Discussion}

These results provide limited evidence for our original hypothesis that sweets and sweetened beverages would be positively associated with risk of pancreatic cancer, although results varied by sex. Total sweets and specific 
Table 3 Odds ratios (OR) and 95\% confidence intervals (CI) for pancreatic cancer and daily servings of sweetened beverages in a populationbased case-control study, San Francisco Bay Area, California

\begin{tabular}{|c|c|c|c|c|c|c|c|c|c|c|c|c|}
\hline \multirow[b]{2}{*}{$\begin{array}{l}\text { Sweetened beverages ( } 1 \text { serving }=1 \text { can, } \\
\text { glass, bottle, or cup) }\end{array}$} & \multicolumn{8}{|c|}{ Men + Women } & \multicolumn{2}{|l|}{ Men } & \multicolumn{2}{|c|}{ Women } \\
\hline & $\begin{array}{l}\text { Case } \\
n\end{array}$ & $\%$ & $\begin{array}{l}\text { Control } \\
n\end{array}$ & $\%$ & $\mathrm{OR}^{\mathrm{a}}$ & $95 \% \mathrm{CI}^{\mathrm{a}}$ & $\mathrm{OR}^{\mathrm{b}}$ & $95 \% \mathrm{CI}^{\mathrm{b}}$ & $\mathrm{OR}^{\mathrm{b}}$ & $95 \% \mathrm{CI}^{\mathrm{b}}$ & $\mathrm{OR}^{\mathrm{b}}$ & $95 \% \mathrm{CI}^{\mathrm{b}}$ \\
\hline \multicolumn{13}{|l|}{ All sweetened beverages/day } \\
\hline 0 & 111 & 21 & 348 & 21 & 1.0 & Ref. & 1.0 & Ref. & 1.0 & Ref. & 1.0 & Ref. \\
\hline$<1$ & 249 & 47 & 942 & 55 & 0.8 & $0.6,1.0$ & 0.7 & $0.6,1.0$ & 1.0 & $0.6,1.5$ & 0.6 & $0.4,0.9$ \\
\hline$\geq 1$ & 166 & 32 & 411 & 24 & 1.1 & $0.8,1.5$ & 1.0 & $0.7,1.3$ & 1.0 & $0.6,1.7$ & 1.0 & $0.6,1.6$ \\
\hline Trend- $p$ & & & & & 0.03 & & 0.7 & & 0.6 & & 0.1 & \\
\hline \multicolumn{13}{|l|}{ Total carbonated beverages/day } \\
\hline 0 & 140 & 27 & 468 & 28 & 1.0 & Ref. & 1.0 & Ref. & 1.0 & Ref. & 1.0 & Ref. \\
\hline$<1$ & 242 & 46 & 894 & 53 & 0.9 & $0.7,1.1$ & 0.8 & $0.6,1.0$ & 1.0 & $0.7,1.6$ & 0.7 & $0.5,1.0$ \\
\hline$\geq 1$ & 144 & 27 & 339 & 20 & 1.3 & $1.0,1.7$ & 1.1 & $0.8,1.5$ & 1.2 & $0.8,2.0$ & 1.1 & $0.7,1.7$ \\
\hline Trend- $p$ & & & & & 0.02 & & 0.6 & & 0.9 & & 0.2 & \\
\hline \multicolumn{13}{|l|}{ Total sugar-type carbonated beverages/day } \\
\hline 0 & 284 & 54 & 961 & 57 & 1.0 & Ref. & 1.0 & Ref. & 1.0 & Ref. & 1.0 & Ref. \\
\hline$<1$ & 190 & 36 & 610 & 36 & 1.0 & $0.8,1.2$ & 1.0 & $0.8,1.2$ & 1.1 & $0.8,1.5$ & 0.8 & $0.6,1.2$ \\
\hline$\geq 1$ & 52 & 10 & 130 & 8 & 1.1 & $0.8,1.6$ & 0.9 & $0.6,1.3$ & 0.9 & $0.5,1.5$ & 0.9 & $0.4,1.8$ \\
\hline Trend- $p$ & & & & & 0.3 & & 0.4 & & 0.3 & & 0.9 & \\
\hline \multicolumn{13}{|l|}{ Total sugar-free carbonated beverages/day } \\
\hline 0 & 310 & 59 & 1,016 & 60 & 1.0 & Ref. & 1.0 & Ref. & 1.0 & Ref. & 1.0 & Ref. \\
\hline$<1$ & 124 & 24 & 487 & 29 & 0.8 & $0.7,1.1$ & 0.8 & $0.7,1.1$ & 1.2 & $0.8,1.7$ & 0.6 & $0.4,1.0$ \\
\hline$\geq 1$ & 92 & 17 & 198 & 12 & 1.5 & $1.2,2.1$ & 1.5 & $1.1,2.1$ & 1.8 & $1.1,2.8$ & 1.4 & $0.9,2.3$ \\
\hline Trend- $p$ & & & & & 0.04 & & 0.2 & & 0.4 & & 0.3 & \\
\hline \multicolumn{13}{|l|}{ Coke, Pepsi, or other cola with sugar } \\
\hline$<1 /$ month & 347 & 66 & 1,175 & 69 & 1.0 & Ref. & 1.0 & Ref. & 1.0 & Ref. & 1.0 & Ref. \\
\hline $1-3 /$ month & 55 & 11 & 179 & 11 & 1.0 & $0.7,1.4$ & 1.0 & $0.7,1.4$ & 1.2 & $0.8,1.8$ & 0.8 & $0.5,1.4$ \\
\hline 1-6/week & 86 & 16 & 247 & 15 & 1.1 & $0.8,1.4$ & 1.1 & $0.8,1.5$ & 1.1 & $0.8,1.7$ & 1.0 & $0.6,1.7$ \\
\hline$\geq 1 /$ day & 38 & 7 & 100 & 6 & 1.1 & $0.7,1.7$ & 0.9 & $0.6,1.4$ & 0.9 & $0.5,1.5$ & 1.0 & $0.5,2.1$ \\
\hline Trend- $p$ & & & & & 0.6 & & 0.99 & & 0.9 & & 0.9 & \\
\hline \multicolumn{13}{|c|}{ Caffeine-free coke, Pepsi, or other cola with sugar } \\
\hline$<1 /$ month & 497 & 94 & 1,598 & 94 & 1.0 & Ref. & 1.0 & Ref. & 1.0 & Ref. & 1.0 & Ref. \\
\hline $1-3 /$ month & 13 & 2 & 55 & 3 & 0.8 & $0.4,1.4$ & 0.9 & $0.5,1.6$ & 0.9 & $0.4,2.0$ & 0.8 & $0.3,2.4$ \\
\hline 1-6/week & 15 & 3 & 37 & 2 & 1.1 & $0.6,2.1$ & 1.0 & $0.6,2.0$ & 1.4 & $0.7,3.0$ & 0.5 & $0.1,1.9$ \\
\hline$\geq 1 /$ day & 1 & 0.2 & 10 & 0.6 & 0.3 & $0.04,2.3$ & 0.3 & $0.04,2.7$ & 0.4 & $0.05,2.3$ & NA & NA \\
\hline Trend- $p$ & & & & & 0.5 & & 0.5 & & 0.96 & & 0.2 & \\
\hline \multicolumn{13}{|l|}{ Other carbonated beverage with sugar } \\
\hline$<1 /$ month & 364 & 69 & 1,229 & 72 & 1.0 & Ref. & 1.0 & Ref. & 1.0 & Ref. & 1.0 & Ref. \\
\hline $1-3 /$ month & 67 & 13 & 200 & 12 & 1.1 & $0.8,1.5$ & 1.1 & $0.8,1.5$ & 1.2 & 0.81 .8 & 0.8 & $0.5,1.4$ \\
\hline $1-6 /$ week & 78 & 15 & 241 & 14 & 1.0 & $0.7,1.3$ & 1.0 & $0.7,1.3$ & 1.0 & $0.6,1.4$ & 1.1 & $0.7,1.8$ \\
\hline$\geq 1 /$ day & 17 & 3 & 31 & 2 & 1.6 & $0.8,2.9$ & 1.2 & $0.6,2.2$ & 1.4 & $0.6,3.1$ & 0.7 & $0.2,2.3$ \\
\hline Trend- $p$ & & & & & 0.5 & & 0.8 & & 0.7 & & 0.8 & \\
\hline \multicolumn{13}{|l|}{ Low calorie cola } \\
\hline$<1 /$ month & 381 & 72 & 1,272 & 75 & 1.0 & Ref. & 1.0 & Ref. & 1.0 & Ref. & 1.0 & Ref. \\
\hline $1-3 /$ month & 34 & 6 & 122 & 7 & 1.0 & $0.6,1.4$ & 1.0 & $0.7,1.5$ & 1.0 & $0.6,1.8$ & 1.0 & $0.5,1.9$ \\
\hline 1-6/week & 54 & 10 & 197 & 12 & 0.9 & $0.7,1.3$ & 0.9 & $0.7,1.3$ & 1.1 & $0.7,1.7$ & 0.8 & $0.4,1.4$ \\
\hline$\geq 1 /$ day & 57 & 11 & 110 & 6 & 1.8 & $1.2,2.5$ & 1.7 & $1.2,2.4$ & 1.8 & $1.1,2.9$ & 1.6 & $0.9,2.8$ \\
\hline Trend- $p$ & & & & & 0.04 & & 0.06 & & 0.06 & & 0.4 & \\
\hline
\end{tabular}


Table 3 continued

\begin{tabular}{|c|c|c|c|c|c|c|c|c|c|c|c|c|}
\hline \multirow[b]{2}{*}{$\begin{array}{l}\text { Sweetened beverages ( } 1 \text { serving }=1 \text { can, } \\
\text { glass, bottle, or cup) }\end{array}$} & \multicolumn{8}{|c|}{ Men + Women } & \multicolumn{2}{|l|}{ Men } & \multicolumn{2}{|c|}{ Women } \\
\hline & $\begin{array}{l}\text { Case } \\
n\end{array}$ & $\%$ & $\begin{array}{l}\text { Control } \\
n\end{array}$ & $\%$ & $\mathrm{OR}^{\mathrm{a}}$ & $95 \% \mathrm{CI}^{\mathrm{a}}$ & $\mathrm{OR}^{\mathrm{b}}$ & $95 \% \mathrm{CI}^{\mathrm{b}}$ & $\mathrm{OR}^{\mathrm{b}}$ & $95 \% \mathrm{CI}^{\mathrm{b}}$ & $\mathrm{OR}^{\mathrm{b}}$ & $95 \% \mathrm{Cl}^{\mathrm{b}}$ \\
\hline \multicolumn{13}{|l|}{ Low calorie caffeine-free cola } \\
\hline$<1 /$ month & 437 & 83 & 1,394 & 82 & 1.0 & Ref. & 1.0 & Ref. & 1.0 & Ref. & 1.0 & Ref. \\
\hline $1-3 /$ month & 24 & 5 & 83 & 5 & 1.0 & $0.6,1.6$ & 1.0 & $0.6,1.6$ & 1.1 & $0.5,2.2$ & 0.9 & $0.5,1.8$ \\
\hline $1-6 /$ week & 43 & 8 & 171 & 10 & 0.8 & $0.6,1.1$ & 0.8 & $0.5,1.1$ & 0.9 & $0.5,1.4$ & 0.7 & $0.4,1.3$ \\
\hline$\geq 1 /$ day & 22 & 4 & 51 & 3 & 1.4 & $0.8,2.4$ & 1.1 & $0.7,2.0$ & 1.1 & $0.5,2.5$ & 1.3 & $0.6,2.7$ \\
\hline Trend- $p$ & & & & & 0.96 & & 0.6 & & 0.9 & & 0.7 & \\
\hline \multicolumn{13}{|l|}{ Other low calorie carbonated beverage } \\
\hline$<1 /$ month & 418 & 79 & 1,366 & 80 & 1.0 & Ref. & 1.0 & Ref. & 1.0 & Ref. & 1.0 & Ref. \\
\hline $1-3 /$ month & 40 & 8 & 128 & 8 & 1.0 & $0.7,1.5$ & 1.0 & $0.7,1.5$ & 1.5 & $0.9,2.5$ & 0.7 & $0.4,1.3$ \\
\hline $1-6 /$ week & 47 & 9 & 167 & 10 & 0.9 & $0.6,1.2$ & 0.9 & $0.6,1.2$ & 1.0 & $0.6,1.6$ & 0.6 & $0.4,1.2$ \\
\hline$\geq 1 /$ day & 21 & 4 & 39 & 2 & 1.6 & $0.9,2.8$ & 1.4 & $0.8,2.5$ & 1.8 & $0.8,3.8$ & 1.2 & $0.4,3.2$ \\
\hline Trend- $p$ & & & & & 0.6 & & 0.97 & & 0.2 & & 0.2 & \\
\hline \multicolumn{13}{|c|}{ Hawaii punch, lemonade, or other non-carbonated fruit drinks } \\
\hline$<1 /$ month & 359 & 68 & 1,075 & 63 & 1.0 & Ref. & 1.0 & Ref. & 1.0 & Ref. & 1.0 & Ref. \\
\hline $1-3 /$ month & 71 & 14 & 286 & 17 & 0.7 & $0.5,1.0$ & 0.7 & $0.5,1.0$ & 0.7 & $0.5,1.1$ & 0.7 & $0.5,1.2$ \\
\hline $1-6 /$ week & 75 & 14 & 292 & 17 & 0.7 & $0.5,0.9$ & 0.7 & $0.5,0.9$ & 0.8 & $0.5,1.2$ & 0.5 & $0.3,0.9$ \\
\hline$\geq 1 /$ day & 21 & 4 & 48 & 3 & 1.1 & $0.6,1.9$ & 1.0 & $0.6,1.8$ & 0.4 & $0.2,1.1$ & 2.0 & $1.0,4.2$ \\
\hline Trend- $p$ & & & & & 0.03 & & 0.03 & & 0.06 & & 0.4 & \\
\hline
\end{tabular}

${ }^{a}$ Adjusted for age, sex, and energy intake (kcal/day, men: quartiles, women: quartiles)

b Additionally adjusted for body mass index $\left(<25.0,25.0-29.9, \geq 30 \mathrm{~kg} / \mathrm{m}^{2}\right.$ ), race (white, black/African American, Asian/Pacific Islander, others), education (<high-school graduate, high-school graduate, 1-4 years college, graduate school), smoking (never smoker, former cigarette smoker who had quit smoking $>15$ years previously, former cigarette smoker who had quit smoking 1-15 years previously, current cigarette smoker or former cigarette smoker who had quit smoking $<1$ year previously, pipe and/or cigar smoker), history of diabetes (yes, no), and physical activity (30-min moderate exercise each time: $<1$ month, 1-4/month, 2-6/week, and daily); Additionally, all sweetened beverages, sugar-type carbonated beverages, and sugar-free carbonated beverages were adjusted for quartiles of red meat, white meat, vegetable and fruit, eggs, fish, dairy, whole grain, and refined grain, and sweets $(0,<1$, and $\geq 1$ serving per day), and were mutually adjusted for each other

forms of candy were linked to greater risk of pancreatic cancer among men, but inconsistently, or not related, among women. The individual sweets that were positively associated with pancreatic cancer risk included sweet condiments, pure chocolate, and other mixed candy bars, but not candy without chocolate. It is possible that saturated fat intake may underlie several of these associations, as we previously reported an increased risk of pancreatic cancer for greater intake of total and saturated fat in this population [20], and pure or mixed chocolate bars are high in fat content. Furthermore, sweet condiments may often be eaten on bread with butter, the latter of which was also strongly positively associated with risk in our prior report [20]. Furthermore, beverages with added sugar and teaspoons of sugar added to food were not linked to greater risk, whereas some sugar-free sodas and low-calorie colas were moderately positively related to risk among men. Consistent with our hypotheses, other non- or low-calorie beverages such as water, tea, or coffee were unassociated with risk.
The positive association for sweets and pancreatic cancer risk among men but not women and the positive association for low-calorie, but not sugar-sweetened, soft drinks are somewhat inconsistent with prior reports, although the literature is limited on this topic. Previous cohort studies have reported associations for sweets and pancreatic cancer risk among men and women combined, or just among women. In the Swedish Mammography Cohort, intake of sugar, soft drinks, and sweetened fruit soups/stew were positively associated with 50-90\% increased risk among women [29]. In the Nurses Health Study and Health Professionals Follow-up Study, soft drinks were modestly positively associated with pancreatic cancer risk among women, but not men [30]. In this twocohort analysis, non-cola diet soft drinks were also associated with a $50 \%$ increase in risk of pancreatic cancer; although confidence estimates included the null [30]. In a distinct large cohort of men and women, fruit and fruit juice intake, but not soda, was positively associated with risk [31]. However, the authors commented that this likely 
Table 4 Odds ratios (OR) and 95\% confidence intervals (CI) for pancreatic cancer and intake of fructose, sucrose, lactose in a population-based case-control study, San Francisco Bay Area, California

\begin{tabular}{|c|c|c|c|c|c|c|c|c|c|c|c|c|}
\hline \multirow[b]{2}{*}{ Quartiles } & \multicolumn{8}{|c|}{ Men + Women } & \multicolumn{2}{|l|}{ Men } & \multicolumn{2}{|c|}{ Women } \\
\hline & Case $n$ & $\%$ & Control $n$ & $\%$ & $\mathrm{OR}^{\mathrm{a}}$ & $95 \% \mathrm{CI}^{\mathrm{a}}$ & $\mathrm{OR}^{\mathrm{b}}$ & $95 \% \mathrm{CI}^{\mathrm{b}}$ & $\mathrm{OR}^{\mathrm{b}}$ & $95 \% \mathrm{CI}^{\mathrm{b}}$ & $\mathrm{OR}^{\mathrm{b}}$ & $95 \% \mathrm{CI}^{\mathrm{b}}$ \\
\hline \multicolumn{13}{|c|}{ Fructose, gm/day } \\
\hline Quartile 1 & 160 & 30 & 426 & 25 & 1.0 & Referent & 1.0 & Referent & 1.0 & Referent & 1.0 & Referent \\
\hline Quartile 2 & 129 & 25 & 424 & 25 & 0.8 & $0.6-1.1$ & 0.9 & $0.7-1.2$ & 1.0 & $0.7-1.4$ & 0.8 & $0.5-1.2$ \\
\hline Quartile 3 & 124 & 24 & 425 & 25 & 0.8 & $0.6-1.0$ & 0.9 & $0.7-1.2$ & 0.8 & $0.6-1.2$ & 0.9 & $0.6-1.4$ \\
\hline Quartile 4 & 112 & 21 & 426 & 25 & 0.7 & $0.5-0.9$ & 0.8 & $0.6-1.1$ & 0.8 & $0.5-1.1$ & 0.9 & $0.6-1.3$ \\
\hline Trend- $p$ & & & & & 0.01 & & 0.2 & & 0.05 & & 0.9 & \\
\hline \multicolumn{13}{|c|}{ Sucrose, gm/day } \\
\hline Quartile 1 & 141 & 27 & 425 & 25 & 1.0 & Referent & 1.0 & Referent & 1.0 & Referent & 1.0 & Referent \\
\hline Quartile 2 & 125 & 24 & 425 & 25 & 0.9 & $0.7-1.2$ & 1.0 & $0.8-1.4$ & 1.1 & $0.8-1.7$ & 0.9 & $0.6-1.5$ \\
\hline Quartile 3 & 121 & 23 & 425 & 25 & 0.9 & $0.7-1.2$ & 1.0 & $0.7-1.3$ & 1.0 & $0.7-1.5$ & 1.2 & $0.8-1.8$ \\
\hline Quartile 4 & 138 & 26 & 426 & 25 & 1.0 & $0.8-1.3$ & 1.0 & $0.8-1.4$ & 1.0 & $0.7-1.5$ & 1.1 & $0.7-1.7$ \\
\hline Trend- $p$ & & & & & 1.0 & & 0.9 & & 1.0 & & 0.6 & \\
\hline \multicolumn{13}{|c|}{ Lactose $^{\mathrm{c}}$, gm/day } \\
\hline Quartile 1 & 94 & 18 & 425 & 25 & 1.0 & Referent & 1.0 & Referent & 1.0 & Referent & 1.0 & Referent \\
\hline Quartile 2 & 136 & 26 & 425 & 25 & 1.5 & $1.1-2.0$ & 1.5 & $1.1-2.0$ & 1.6 & $1.1-2.7$ & 1.2 & $0.7-1.9$ \\
\hline Quartile 3 & 131 & 25 & 425 & 25 & 1.4 & $1.1-1.9$ & 1.6 & $1.2-2.1$ & 1.8 & $1.2-2.8$ & 2.0 & $1.3-3.1$ \\
\hline Quartile 4 & 164 & 31 & 425 & 25 & 1.8 & $1.3-2.4$ & 2.0 & $1.5-2.7$ & 2.1 & $1.3-3.0$ & 1.7 & $1.1-2.7$ \\
\hline Trend- $p$ & & & & & 0.0003 & & $<0.0001$ & & 0.003 & & 0.03 & \\
\hline
\end{tabular}

${ }^{a}$ Used energy residual model; adjusted for age, sex, and energy intake (kcal/day, men: quartiles, women: quartiles)

b Additionally adjusted for body mass index $\left(<25.0,25.0-29.9, \geq 30 \mathrm{~kg} / \mathrm{m}^{2}\right)$, race (white, black/African American, Asian/Pacific Islander, others), education ( $<$ high-school graduate, high-school graduate, 1-4 years college, graduate school), smoking (never smoker, former cigarette smoker who had quit smoking $>15$ years previously, former cigarette smoker who had quit smoking 1-15 years previously, current cigarette smoker or former cigarette smoker who had quit smoking $<1$ year previously, pipe and/or cigar smoker), history of diabetes (yes, no), and physical activity (30-min moderate exercise each time: $<1$ month, 1-4/month, 2-6/week, and daily)

c Additionally adjusted for total fat consumption by quartiles

reflected an overall positive association for fructose/sugars that were strongly correlated with fruits and juices, and that there may have been measurement error in the assessment of sodas. In contrast, the large prospective National Institutes of Health (NIH)-AARP Diet and Health Study reported no association for high intake of total added sugar, sugar-sweetened foods and beverages, or diet soft drinks and pancreatic cancer [32].

The trend toward stronger associations for sweets being more common among those who were obese or exercised infrequently is consistent with results from the Nurses Health Study, where positive associations for sweetened soft-drink intake [30] and fructose [33] and pancreatic cancer risk were stronger among women with a high BMI or who exercised less. Similarly, in the NIH-AARP cohort, there were suggestive (but not always statistically significant) positive trends for total added sugar and pancreatic cancer risk only among those who were obese or less frequent exercisers [32].

Data from case-control studies are also limited, but one previous case-control study reported a non-statistically significant $50 \%$ increased risk for sweet foods [34], while another observed a statistically significant $80 \%$ elevation in risk associated with dessert intake among women and no association among men [35]. In contrast to our results, an early case-control study that examined a wide range of individual dietary factors reported an inverse association for consumption of diet soda and risk of pancreatic cancer [36]. It is possible that comparison of these early results with our study is inappropriate given the dramatic changes in diet soda consumption patterns over the last several decades.

Another way to examine the impact of sweets on pancreatic cancer risk has been to examine intake of different sugars. Similar to our study, four case-control studies and one cohort have reported no association for simple sugars $[35,37]$ or sucrose consumption [37-40]; although two of these reported statistically significant doubling to tripling of risk for added or refined sugar intake [38, 40]. Polysaccharides but not mono- or disaccharides were related to a 2.6-fold increase in risk in a case-control study [34]. In contrast to our findings, in the large Hawaii-Los Angeles 
Multiethnic Cohort, risk increased with greater intake of total sugars, fructose, and sucrose, and was statistically significant for fructose (relative risk $=1.4 ; 95 \% \mathrm{CI}: 1.0$, 1.8; for highest vs. lowest quartiles) [31]. In the Nurses Health Study, fructose was positively associated with pancreatic cancer risk among overweight women with low physical activity levels (fructose relative risk $=3.2,95 \%$ CI: $1.1,8.9$ when comparing extreme quartiles).

Lactose is a sugar naturally occurring in milk and milk products, and there are very limited prior reports on lactose and pancreatic cancer. Baghurst et al. observed no association for lactose and risk of pancreatic cancer in an earlier case-control study [38]. Milk and dairy intake have not been consistently associated with pancreatic cancer [41], with most studies reporting null associations [34, 35, 40-47], and only a few observing positive $[20,48]$ or inverse associations [49]. In a study of cases only, Morales et al. reported that daily versus non-daily intake of dairy products, but not other food groups, was associated with a five-fold greater occurrence of K-Ras mutated pancreatic tumors, and K-ras mutations are considered to be an early event in pancreatic carcinogenesis [50]. We observed a positive association for lactose and pancreatic cancer risk, independent of total energy and fat; consistent with and expanding on the previously reported positive association for dairy products and pancreatic cancer risk in this population [20],

The observed null results for coffee and pancreatic cancer risk were consistent with several previous studies [36, 42, 48, 49, 51-54]. The slightly elevated risk for nonherbal tea among men in our study may be due to chance, and was contrary to several other null reports [36, 42, 53]. We did not directly examine sweetened tea or coffee; however, teaspoons of added sugar to foods or beverages and artificial sweeteners were unassociated with risk.

Simple carbohydrates (e.g., sugars) raise blood glucose levels more than starches and other macronutrients $[55,56]$. Chronic high intake of sweets may increase pancreatic cancer risk by affecting glucose metabolism and predisposing to hyperglycemia, insulin insensitivity, and hyperinsulinemia. Glycemic load and index are measures of the body's glucoseresponse (or insulin demand) to carbohydrate intake. Six cohort studies examining estimated glycemic load or index from self-reported questionnaires and pancreatic cancer risk observed no associations [31, 37, 57-59], while the Nurses Health Study reported that glycemic load was strongly related to risk among overweight or sedentary women [33].

In contrast, prospective studies have linked higher circulating fasting glucose [4-7] and insulin levels [4] to future risk of pancreatic cancer many years later. The Chicago Heart Association Detection Project, with an average of 25 years of follow-up examined fasting glucose and pancreatic cancer mortality. Their results for fasting glucose and risk of pancreatic cancer were similar even when they excluded cases that occurred within the first five years of follow-up [5]. In the Alpha Tocopherol Beta-Carotene Cancer Prevention trial, associations for fasting glucose and pancreatic cancer were stronger when limited only to those participants with more than 10 years of follow-up [4]. Taken together, these studies support a positive dose-dependent relationship between hyperglycemia and pancreatic cancer incidence or mortality. The lack of consistent association between sweet foods or sweetened beverages and pancreatic cancer risk in this study may in part be due to the variable effects of sugar intake on glucose metabolism, depending on sugar type, fiber, protein, fat and starch composition, temperature, cooking, and processing of the food [55].

We cannot exclude the possibility of chance to explain some of our results. The elevated risk observed in the current study for total and specific sweets, in particular among those who exercised less, had a high BMI, or smoked, provides some support for the hypothesis that sweets intake plus lifestyle factors combined may predispose to impaired glucose tolerance that can subsequently influence pancreatic cancer risk. Impaired glucose tolerance is positively correlated with high glucose intake, obesity, sedentary habits, and smoking [33, 60-62]. The elevated risks associated with diet soda intake, in particular among men, those who were obese, did not have diabetes, or who were smokers, could possibly be due to residual confounding by other unmeasured diet and lifestyle factors.

This study had several possible limitations that must be considered. Recall bias may have affected the results, although, when this study was conducted little was known about dietary risk factors for pancreatic cancer. Non-differential measurement error in assessment of the dietary exposures could have led to a bias toward the null, although prior publications from this population reported positive associations for specific meats and fats, and inverse associations for fruit and vegetable intake, consistent with other cohort and case-control studies [11, 20, 41]. We were unable to examine the effects of glycemic load or index or pre-diagnostic circulating glucose on risk of pancreatic cancer in this study. There was a $67 \%$ response rate among eligible cases and controls. It is possible that eligible control subjects who chose to participate were those who tended to be more health conscious and thus different than the population that gave rise to the cases. There is also the possibility of survivor bias, whereby those cases who did not survive long enough to be interviewed may have differed meaningfully in their diets compared to those who were included.

Strengths of this study include the population-based design, large sample size, detailed validated dietary questionnaire completed using only direct in-person interviews, and no proxy interviews. We interviewed participants about dietary practices one year before their cancer diagnosis or interview for controls to avoid recent assessment of dietary 
changes due to pancreatic cancer. The refusal rate was low at $8 \%$, and the primary reason we lost patients was the rapid mortality rate. In conclusion, these data provide limited evidence for our original hypotheses that sweets, sugars, and sweetened beverages increase the risk of pancreatic cancer. The stronger associations stratified by sex, BMI, exercise, diabetes status, and smoking were intriguing and warrant further research.

Acknowledgments The collection of cancer incidence data used in this study was supported by the California Department of Public Health as part of the statewide cancer reporting program mandated by California Health and Safety Code Sect. 103885; the National Cancer Institute's Surveillance, Epidemiology and End Results Program under contract N01-PC-35136 awarded to the Northern California Cancer Center, contract N01-PC-35139 awarded to the University of Southern California, and contract N02-PC-15105 awarded to the Public Health Institute; and the Centers for Disease Control and Prevention's National Program of Cancer Registries, under agreement \#U55/CCR921930-02 awarded to the Public Health Institute. The ideas and opinions expressed herein are those of the author(s) and endorsement by the State of California, Department of Public Health the National Cancer Institute, and the Centers for Disease Control and Prevention or their Contractors and Subcontractors is not intended nor should be inferred.

Grant support National Institutes of Health, NCI grants CA59706, CA89726, CA72712, CA09889, CA108370 (E. A. Holly, Principal Investigator) and the Rombauer Pancreatic Cancer Research Fund.

Open Access This article is distributed under the terms of the Creative Commons Attribution Noncommercial License which permits any noncommercial use, distribution, and reproduction in any medium, provided the original author(s) and source are credited.

\section{References}

1. American Cancer Society. Cancer facts and figures-2008. Atlanta, GA: American Cancer Society; 2008 Contract No.: Document Number

2. Huxley R, Ansary-Moghaddam A, Berrington de Gonzalez A, Barzi F, Woodward M (2005) Type-II diabetes and pancreatic cancer: a meta-analysis of 36 studies. Br J Cancer 92(11):20762083. doi:10.1038/sj.bjc.6602619

3. Stevens RJ, Roddam AW, Beral V (2007) Pancreatic cancer in type 1 and young-onset diabetes: systematic review and metaanalysis. Br J Cancer 96(3):507-509. doi:10.1038/sj.bjc.6603571

4. Stolzenberg-Solomon RZ, Graubard BI, Chari S, Limburg P, Taylor PR, Virtamo J et al (2005) Insulin, glucose, insulin resistance, and pancreatic cancer in male smokers. JAMA 294(22):2872-2878. doi:10.1001/jama.294.22.2872

5. Gapstur SM, Gann PH, Lowe W, Liu K, Colangelo L, Dyer A (2000) Abnormal glucose metabolism and pancreatic cancer mortality. JAMA 283(19):2552-2558. doi:10.1001/jama.283.19. 2552

6. Jee SH, Ohrr H, Sull JW, Yun JE, Ji M, Samet JM (2005) Fasting serum glucose level and cancer risk in Korean men and women. JAMA 293(2):194-202. doi:10.1001/jama.293.2.194

7. Yun JE, Jo I, Park J, Kim MT, Ryu HG, Odongua N et al (2006) Cigarette smoking, elevated fasting serum glucose, and risk of pancreatic cancer in Korean men. Int J Cancer 119(1):208-212. doi:10.1002/ijc. 21816

8. Michaud DS, Wolpin B, Giovannucci E, Liu S, Cochrane B, Manson JE et al (2007) Prediagnostic plasma C-peptide and pancreatic cancer risk in men and women. Cancer Epidemiol Biomarkers Prev 16(10):2101-2109. doi:10.1158/1055-9965.EPI-07-0182

9. Hennig R, Ding XZ, Adrian TE (2004) On the role of the islets of Langerhans in pancreatic cancer. Histol Histopathol 19(3):9991011

10. Duell EJ, Holly EA, Bracci PM, Liu M, Wiencke JK, Kelsey KT (2002) A population-based, case-control study of polymorphisms in carcinogen-metabolizing genes, smoking, and pancreatic adenocarcinoma risk. J Natl Cancer Inst 94(4):297-306

11. Chan JM, Wang F, Holly EA (2005) Vegetable and fruit intake and pancreatic cancer in a population-based case-control study in the San Francisco bay area. Cancer Epidemiol Biomarkers Prev 14(9):2093-2097. doi:10.1158/1055-9965.EPI-05-0226

12. Eberle CA, Bracci PM, Holly EA (2005) Anthropometric factors and pancreatic cancer in a population-based case-control study in the San Francisco Bay area. Cancer Causes Control 16(10):12351244. doi:10.1007/s10552-005-0354-y

13. Duell EJ, Holly EA (2005) Reproductive and menstrual risk factors for pancreatic cancer: a population-based study of San Francisco Bay Area women. Am J Epidemiol 161(8):741-747. doi:10.1093/aje/kwi104

14. Holly EA, Chaliha I, Bracci PM, Gautam M (2004) Signs and symptoms of pancreatic cancer: a population-based case-control study in the San Francisco Bay area. Clin Gastroenterol Hepatol 2(6):510-517. doi:10.1016/S1542-3565(04)00171-5

15. Holly EA, Eberle CA, Bracci PM (2003) Prior history of allergies and pancreatic cancer in the San Francisco Bay area. Am J Epidemiol 158(5):432-441. doi:10.1093/aje/kwg174

16. Duell EJ, Holly EA, Bracci PM, Wiencke JK, Kelsey KT (2002) A population-based study of the Arg399Gln polymorphism in Xray repair cross-complementing group 1 (XRCC1) and risk of pancreatic adenocarcinoma. Cancer Res 62(16):4630-4636

17. Slebos RJ, Hoppin JA, Tolbert PE, Holly EA, Brock JW, Zhang RH et al (2000) K-ras and p53 in pancreatic cancer: association with medical history, histopathology, and environmental exposures in a population-based study. Cancer Epidemiol Biomarkers Prev 9(11): 1223-1232

18. Hoppin JA, Tolbert PE, Holly EA, Brock JW, Korrick SA, Altshul LM et al (2000) Pancreatic cancer and serum organochlorine levels. Cancer Epidemiol Biomarkers Prev 9(2):199-205

19. Chan JM, Wang F, Holly EA (2007) Whole grains and risk of pancreatic cancer in a large population-based case-control study in the San Francisco Bay Area, California. Am J Epidemiol 166(10):1174-1185. doi:10.1093/aje/kwm194

20. Chan JM, Wang F, Holly EA (2007) Pancreatic cancer, animal protein and dietary fat in a population-based study, San Francisco Bay Area, California. Cancer Causes Control 18(10):1153-1167. doi:10.1007/s10552-007-9054-0

21. Ko AH, Wang F, Holly EA (2007) Pancreatic cancer and medical history in a population-based case-control study in the San Francisco Bay Area, California. Cancer Causes Control 18(8):809-819. doi:10.1007/s10552-007-9024-6

22. Wang F, Gupta S, Holly EA (2006) Diabetes mellitus and pancreatic cancer in a population-based case-control study in the San Francisco Bay Area, California. Cancer Epidemiol Biomarkers Prev 15(8):1458-1463. doi:10.1158/1055-9965.EPI-06-0188

23. Duell EJ, Casella DP, Burk RD, Kelsey KT, Holly EA (2006) Inflammation, genetic polymorphisms in proinflammatory genes TNF-A, RANTES, and CCR5, and risk of pancreatic adenocarcinoma. Cancer Epidemiol Biomarkers Prev 15(4):726-731. doi: 10.1158/1055-9965.EPI-05-0797 
24. Holmes MD, Powell IJ, Campos H, Stampfer MJ, Giovannucci EL, Willett WC (2007) Validation of a food frequency questionnaire measurement of selected nutrients using biological markers in African-American men. Eur J Clin Nutr 61(11):13281336. doi:10.1038/sj.ejen.1602641

25. Longnecker MP, Lissner L, Holden JM, Flack VF, Taylor PR, Stampfer MJ et al (1993) The reproducibility and validity of a self-administered semiquantitative food frequency questionnaire in subjects from South Dakota and Wyoming. Epidemiology 4(4):356-365. doi:10.1097/00001648-199307000-00012

26. Subar AF, Thompson FE, Kipnis V, Midthune D, Hurwitz P, McNutt $S$ et al (2001) Comparative validation of the Block, Willett and National Cancer Institute food frequency questionnaires : the Eating at America's Table Study. Am J Epidemiol 154(12):1089-1099. doi:10.1093/aje/154.12.1089

27. Salvini S, Hunter DJ, Sampson L, Stampfer MJ, Colditz GA, Rosner $\mathrm{B}$ et al (1989) Food-based validation of a dietary questionnaire: the effects of week-to-week variation in food consumption. Int $\mathrm{J}$ Epidemiol 18(4):858-867. doi:10.1093/ije/18.4.858

28. Willett WC (1990) Nutritional epidemiology. Oxford University Press, New York

29. Larsson SC, Bergkvist L, Wolk A (2006) Consumption of sugar and sugar-sweetened foods and the risk of pancreatic cancer in a prospective study. Am J Clin Nutr 84(5):1171-1176

30. Schernhammer ES, Hu FB, Giovannucci E, Michaud DS, Colditz GA, Stampfer MJ et al (2005) Sugar-sweetened soft drink consumption and risk of pancreatic cancer in two prospective cohorts. Cancer Epidemiol Biomarkers Prev 14(9):2098-2105. doi:10.1158/1055-9965.EPI-05-0059

31. Nothlings U, Murphy SP, Wilkens LR, Henderson BE, Kolonel LN (2007) Dietary glycemic load, added sugars, and carbohydrates as risk factors for pancreatic cancer: the Multiethnic Cohort Study. Am J Clin Nutr 86(5):1495-1501

32. Bao Y, Stolzenberg-Solomon R, Jiao L, Silverman DT, Subar AF, Park Y et al (2008) Added sugar and sugar-sweetened foods and beverages and the risk of pancreatic cancer in the National Institutes of Health-AARP Diet and Health Study. Am J Clin Nutr 88(2):431-440

33. Michaud DS, Liu S, Giovannucci E, Willett WC, Colditz GA, Fuchs CS (2002) Dietary sugar, glycemic load, and pancreatic cancer risk in a prospective study. J Natl Cancer Inst 94(17): 1293-1300

34. Bueno de Mesquita HB, Maisonneuve P, Runia S, Moerman CJ (1991) Intake of foods and nutrients and cancer of the exocrine pancreas: a population-based case-control study in The Netherlands. Int J Cancer 48(4):540-549. doi:10.1002/ijc.2910480411

35. Silverman DT, Swanson CA, Gridley G, Wacholder S, Greenberg RS, Brown LM et al (1998) Dietary and nutritional factors and pancreatic cancer: a case-control study based on direct interviews. J Natl Cancer Inst 90(22):1710-1719. doi:10.1093/jnci/ 90.22.1710

36. Gold EB, Gordis L, Diener MD, Seltser R, Boitnott JK, Bynum TE et al (1985) Diet and other risk factors for cancer of the pancreas. Cancer 55(2):460-467. doi:10.1002/1097-0142(1985 0115)55:2<460::AID-CNCR2820550229>3.0.CO;2-V

37. Heinen MM, Verhage BA, Lumey L, Brants HA, Goldbohm RA, van den Brandt PA (2008) Glycemic load, glycemic index, and pancreatic cancer risk in the Netherlands Cohort Study. Am J Clin Nutr 87(4):970-977

38. Baghurst PA, McMichael AJ, Slavotinek AH, Baghurst KI, Boyle P, Walker AM (1991) A case-control study of diet and cancer of the pancreas. Am J Epidemiol 134(2):167-179

39. Kalapothaki V, Tzonou A, Hsieh CC, Karakatsani A, Trichopoulou A, Toupadaki N et al (1993) Nutrient intake and cancer of the pancreas: a case-control study in Athens, Greece. Cancer Causes Control 4(4):383-389. doi:10.1007/BF00051342
40. Lyon JL, Slattery ML, Mahoney AW, Robison LM (1993) Dietary intake as a risk factor for cancer of the exocrine pancreas. Cancer Epidemiol Biomarkers Prev 2(6):513-518

41. Anderson K, Mack T, Silverman D (2006) Cancer of the pancreas. In: Schottenfeld D, Fraumeni J (eds) Cancer epidemiology and prevention. Oxford University Press, New York, pp 721-763

42. Stolzenberg-Solomon RZ, Pietinen P, Taylor PR, Virtamo J, Albanes D (2002) Prospective study of diet and pancreatic cancer in male smokers. Am J Epidemiol 155(9):783-792. doi:10.1093/ aje/155.9.783

43. La Vecchia C, Negri E, D’Avanzo B, Ferraroni M, Gramenzi A, Savoldelli R et al (1990) Medical history, diet and pancreatic cancer. Oncology 47(6):463-466

44. Michaud DS, Giovannucci E, Willett WC, Colditz GA, Fuchs CS (2003) Dietary meat, dairy products, fat, and cholesterol and pancreatic cancer risk in a prospective study. Am J Epidemiol 157(12):1115-1125. doi:10.1093/aje/kwg098

45. Nothlings U, Wilkens LR, Murphy SP, Hankin JH, Henderson BE, Kolonel LN (2005) Meat and fat intake as risk factors for pancreatic cancer: the multiethnic cohort study. J Natl Cancer Inst 97(19): 1458-1465

46. Soler M, Chatenoud L, La Vecchia C, Franceschi S, Negri E (1998) Diet, alcohol, coffee and pancreatic cancer: final results from an Italian study. Eur J Cancer Prev 7(6):455-460. doi: 10.1097/00008469-199812000-00005

47. Voirol M, Infante F, Raymond L, Hollenweger V, Zurkirch MC, Tuyns A et al (1987) Nutritional profile of patients with cancer of the pancreas. Schweiz Med Wochenschr 117(29):1101-1104

48. Falk RT, Pickle LW, Fontham ET, Correa P, Fraumeni JF Jr (1988) Life-style risk factors for pancreatic cancer in Louisiana: a case-control study. Am J Epidemiol 128(2):324-336

49. Mizuno S, Watanabe S, Nakamura K, Omata M, Oguchi H, Ohashi K et al (1992) A multi-institute case-control study on the risk factors of developing pancreatic cancer. Jpn J Clin Oncol 22(4):286-291

50. Morales E, Porta M, Vioque J, Lopez T, Mendez MA, Pumarega $\mathrm{J}$ et al (2007) Food and nutrient intakes and K-ras mutations in exocrine pancreatic cancer. J Epidemiol Community Health 61(7):641-649. doi:10.1136/jech.2007.060632

51. Mills PK, Beeson WL, Abbey DE, Fraser GE, Phillips RL (1988) Dietary habits and past medical history as related to fatal pancreas cancer risk among Adventists. Cancer 61(12):2578-2585. doi:10.1002/1097-0142(19880615)61:12<2578::AID-CNCR2820 $611232>3.0 . \mathrm{CO} ; 2-0$

52. Norell SE, Ahlbom A, Erwald R, Jacobson G, Lindberg-Navier I, Olin R et al (1986) Diet and pancreatic cancer: a case-control study. Am J Epidemiol 124(6):894-902

53. Shibata A, Mack TM, Paganini-Hill A, Ross RK, Henderson BE (1994) A prospective study of pancreatic cancer in the elderly. Int J Cancer 58(1):46-49. doi:10.1002/ijc.2910580109

54. Zheng W, McLaughlin JK, Gridley G, Bjelke E, Schuman LM, Silverman DT et al (1993) A cohort study of smoking, alcohol consumption, and dietary factors for pancreatic cancer (United States). Cancer Causes Control 4(5):477-482. doi:10.1007/BF00050867

55. Linder MC (1991) Nutrition and metabolism of carbohydrates. In: Linder MC (ed) Nutritional biochemistry and metabolism, 2nd edn. Appleton \& Lange, East Norwalk, pp 21-50

56. Anderson JW (1999) Nutritional management of diabetes mellitus. In: Shils ME, Olson JA, Shike M, Ross CA (eds) Modern nutrition in health and disease, 9th edn. Williams and Wilkins, Philadelphia, pp 1365-1394

57. Johnson KJ, Anderson KE, Harnack L, Hong CP, Folsom AR (2005) No association between dietary glycemic index or load and pancreatic cancer incidence in postmenopausal women. Cancer Epidemiol Biomarkers Prev 14(6):1574-1575. doi:10.1158/10559965.EPI-05-0138 
58. Patel AV, McCullough ML, Pavluck AL, Jacobs EJ, Thun MJ, Calle EE (2007) Glycemic load, glycemic index, and carbohydrate intake in relation to pancreatic cancer risk in a large US cohort. Cancer Causes Control 18(3):287-294. doi:10.1007/ s10552-006-0081-z

59. Silvera SA, Rohan TE, Jain M, Terry PD, Howe GR, Miller AB (2005) Glycemic index, glycemic load, and pancreatic cancer risk (Canada). Cancer Causes Control 16(4):431-436. doi:10.1007/ s10552-004-5028-7

60. Berentzen T, Petersen L, Pedersen O, Black E, Astrup A, Sorensen TI (2007) Long-term effects of leisure time physical activity on risk of insulin resistance and impaired glucose tolerance, allowing for body weight history, in Danish men. Diabet Med 24(1):63-72. doi:10.1111/j.1464-5491.2007.01991.x

61. Mozaffarian D, Marfisi R, Levantesi G, Silletta MG, Tavazzi L, Tognoni $G$ et al (2007) Incidence of new-onset diabetes and impaired fasting glucose in patients with recent myocardial infarction and the effect of clinical and lifestyle risk factors. Lancet 370(9588):667-675. doi:10.1016/S0140-6736(07)61343-9

62. Todoroki I, Shinchi K, Kono S, Imanishi K (1994) Lifestyle and glucose tolerance: a cross-sectional study of Japanese men. Ann Epidemiol 4(5):363-368 